\title{
Advanced product design principles applied for developing a reconfigurable multi-station welding workbench
}

\author{
Vasile Merticaru*, Alexandru-Catalin Paraschiv and Marius-Ionut Ripanu \\ "Gheorghe Asachi" Technical University of Iasi, Romania
}

\begin{abstract}
The research results presented in the paper are part of a larger approach concerning the improvement of product design process sustainability by enhancing the effectiveness and efficiency of Product Design activities. As case study, an applied educational project is exemplified as result sample, particularly referring to applying such advanced design principles in developing a reconfigurable multi-station welding workbench, intended to solve the problem of the lack of space which usually most of the welding workshops have to confront with.
\end{abstract}

\section{Introduction. Problem statement}

There is a good while since the concept and principles of Agile Manufacturing have become a valuable strategic philosophy for industrial production survival and development within a high frequency changing market, [1], bringing fundamental and irreversible structural changes in mastering the uncertainty and redefining the mass production bases, where Flexible Manufacturing only is not any more able to solve the complex competition problems.

In order to deal with the continuously increasing product variety and individualization, Mass Customization has come to replace Mass Production as industrial strategy, [2], and Reconfigurable Manufacturing Systems are nowadays bringing more effective responsiveness to renewing circumstances, being designed for rapidly adjustable capabilities, in terms of functionality and production capacity, [3]. The essence in this direction is represented by modularity and by changing or rearranging the system components.

On the other hand, Sustainability of Product and Process Development is another major theme for advanced industrial and manufacturing engineering research, a lot of new concepts, principles and strategies evolving also in this direction.

But some of the principles of reconfiguration, as they are nominated for integrated manufacturing systems in large production enterprises, [4], are sometimes useful and effective also for product or equipment design for smaller industrials or even for manufacturing workshops, in order to enhance their flexibility and sustainability. We hereby talk about principles such as modularity, scalability, convertibility and not only.

\footnotetext{
*Corresponding author: merticaru@tcm.tuiasi.ro
} 
The particular research results presented further on in the paper are referring to such a problem of integrating and applying some advanced design principles within product and process development activities. The research approach is part of a larger approach concerning the improvement of product design process sustainability by enhancing the effectiveness and efficiency of Product Design activities, by improving the product flexibility, functionality and reliability, by reducing the resources consumption and, not at last, by reducing the product lifecycle environmental impact, all of these being based on integrating some advanced Product Design principles, theories and tools, [5-8].

\section{Research approach substantiation. Conceptual models}

Within the particular research approach being discussed in the paper, a conceptual model has been first of all structured, based on a conceptual analysis, and it is hereby proposed in Fig. 1, referring to the importance of product specifications correctly defining, as core condition for providing product development sustainability.

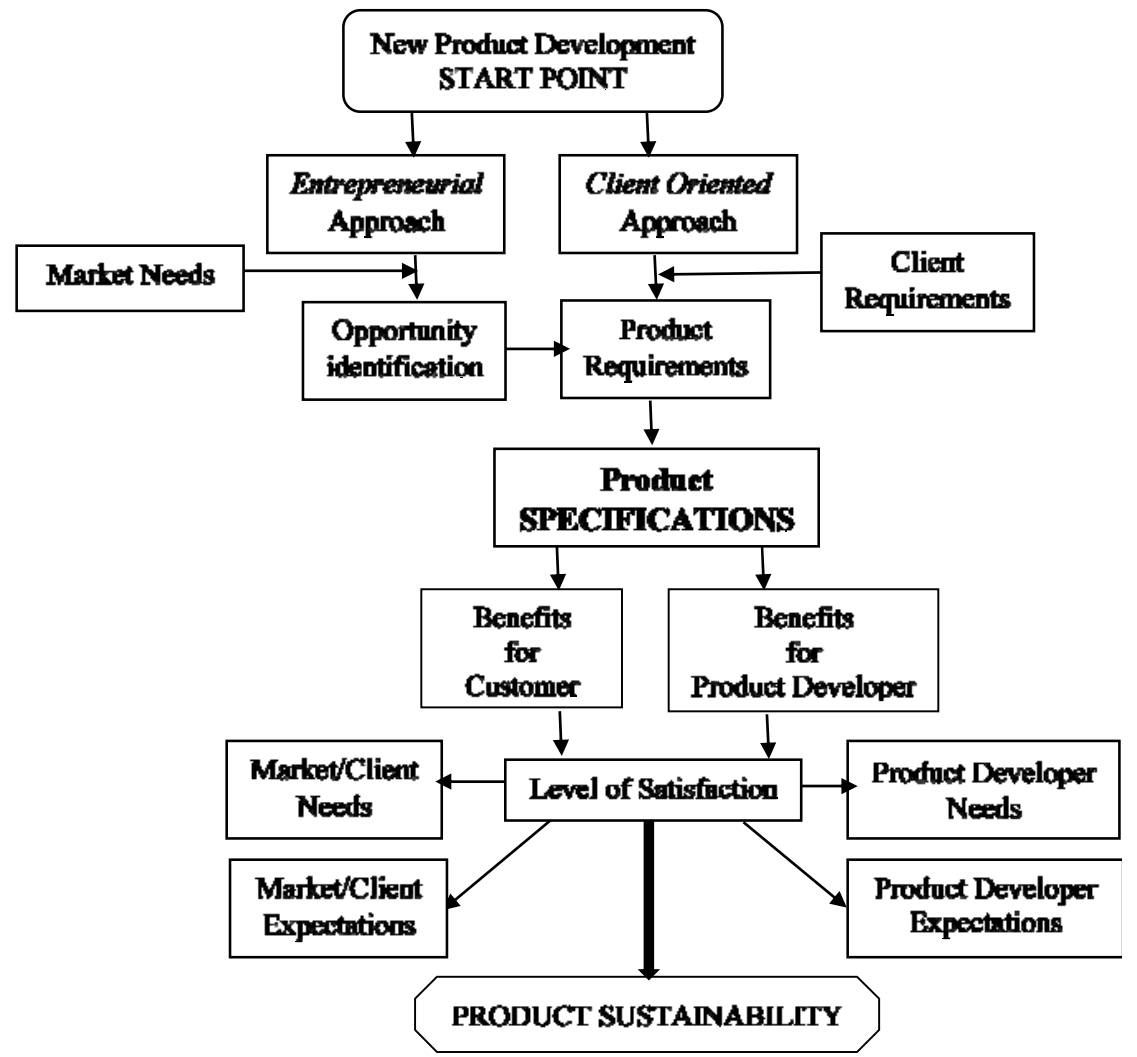

Fig. 1. Conceptual model of product specifications defining for sustainable product development.

Another conceptual model has been further on developed and is proposed in Fig.2, where some ideas related to the principles of Reconfigurable Product Design and Modular Product Design are mainly considered, starting from the need to provide easiness in change for the product or equipment physical structure and functionality, [4], and from the fact that modularity in product structure helps a lot in this direction by the possibility of components rearrangement or change, [3]. 


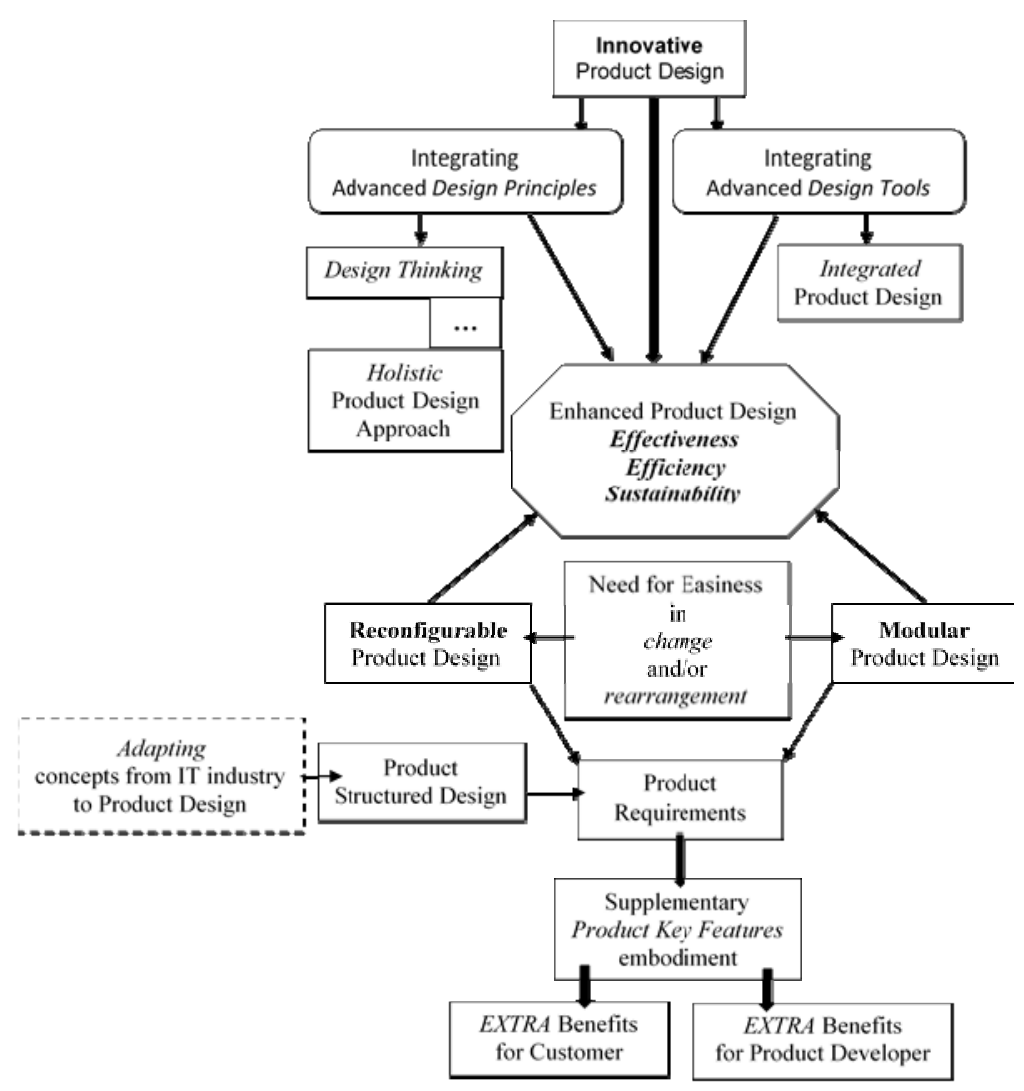

Fig. 2. Conceptual model for convergent integration of advanced design principles within Reconfigurable Product Design.

More than that, the idea of adapting some concepts from IT industry to Product Design activities, have determined to consider also some discussions upon Product Structured Design, [9], within the above mentioned model.

As start point in innovative Product Development, the idea that product conceptualisation must respond to a particular production need identified as an opportunity, [10], has been considered.

Further on, a set of general product requirements and also a specific one have been considered to be identified, in relation to all the advanced design principles included in the above model, Design Thinking, [11], and Holistic Design, [12], being among them.

Some of the identified and further on considered general product requirements are as following: reconfigurable structure; modular structure; product decomposition principles embedment; enhanced features and functionality; layout space optimization (compact design); durable construction; modules reusability for further applications development; ergonomic principles embedment; easy to assemble, install and relocate; easiness in access and use; safety in use; reduced material resource consumption (dematerialization principle); reduced carbon footprint (product lifecycle environmental impact); cleaner work environment.

All the above presented requirements will provide key features for product sustainability, which will become competitive advantages for it. 


\section{Case study. Product design approach and results}

As case study, an applied educational project is exemplified as result sample, particularly referring to applying such advanced design principles in developing a reconfigurable multistation welding workbench, intended to solve the problem of the lack of space which usually most of the welding workshops have to confront with, [13].

Advanced capabilities of Solid Edge as CAD solution, [14], referring mainly to the performances of providing dynamic generation of product model structure and to the advanced parameterizing functions have been used within the case study.

By combining innovation tools and simplicity considerations, the structure of the reconfigurable multi-station welding workbench has been conceived based on two types of main functional modules which can be combined, rearranged and eventually changed for obtaining configurations able to respond to different particular needs of a welding workshop, dimensioned for four up to ten individual working stations. The designed solution also provides optimized spaces for equipment and personal stuff storage.

The main functional module no.1, shown in Fig.3, provides two individual working stations endowed with equipment for welding, cutting and drilling operations, welding gas recipients, fixing devices and additional storage spaces. The module is designed to be combined at least two of them in tandem, for integrating four welding equipment of invertor type, in order to support WIG, MIG/MAG welding processes and also electric arc welding processes using coated electrodes. The modular structure of this module, consisting of a frame and of some auxiliary components, is shown in Fig. 4.

The solution of steel profiles framework, built to withstand the hard conditions of production environment, provides enhanced durability for the mechanical construction.

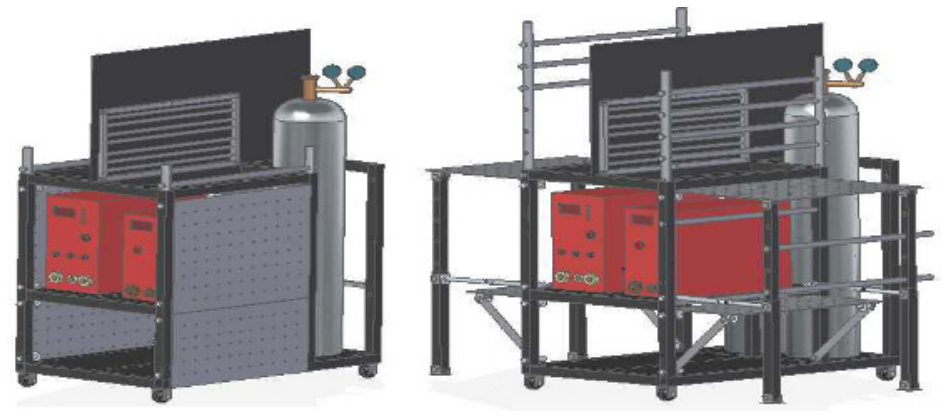

Fig. 3. Folded and unfolded view of main functional module no.1.
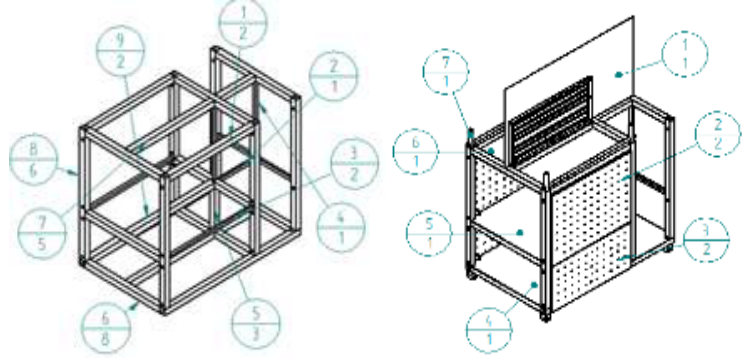

Fig. 4. Modular structure (frame and auxiliary components) of main module no.1

The main functional module no.2, shown in Fig.5, provides a welding fume extraction hood, two foldable tables for creating two additional working stations, two lockers and an auxiliary storage space. The modular structure of this module, consisting also of a frame and of some auxiliary components, is shown in Fig. 6. 


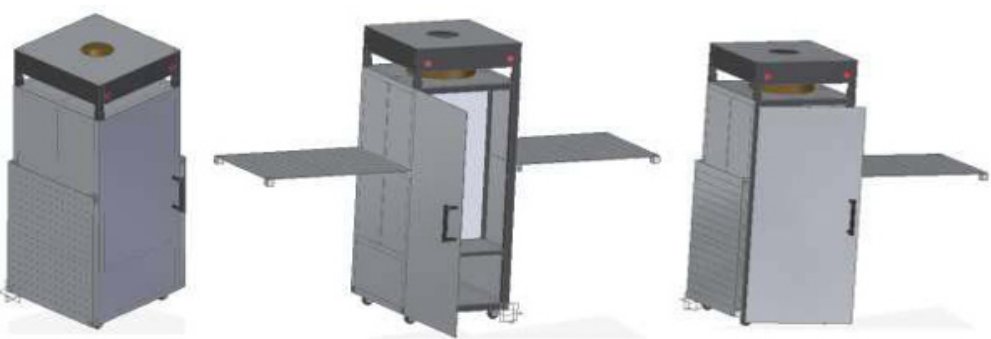

Fig. 5. Folded, unfolded and half-folded views of main module no. 2.
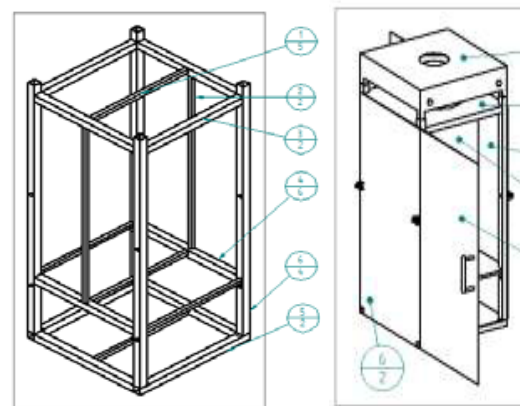

Fig. 6. Modular structure (frame and auxiliary components) of main module no. 2 .

In Fig.7 and Fig.8, two particularly designed configurations, respectively those for four and for ten individual working stations are shown.
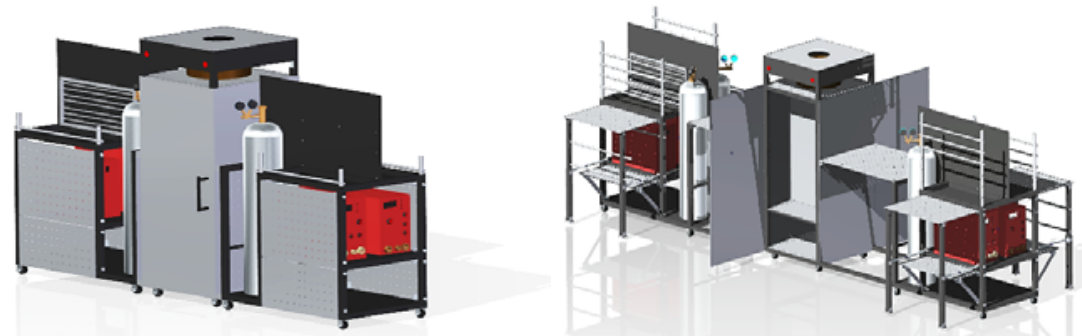

Fig. 7. Folded and unfolded view of configuration with four working stations.

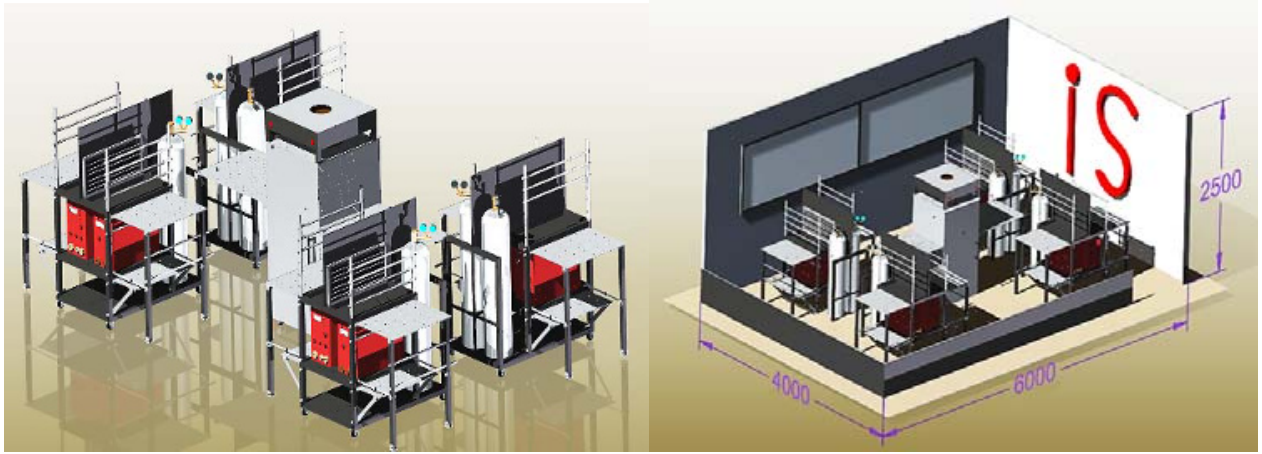

Fig. 8. Unfolded view and workshop layout view of configuration with ten working stations. 


\section{Conclusions}

As a conclusion, we can firstly say that all the above presented conceptual considerations and the results of the case study come to sustain the great importance of appropriately identifying, selecting, combining and aligning the best options to the needs, within Product Design and Product Development activities, starting with an analytic and detailed establishment of product requirements and with their translation into product specifications.

Some general product requirements intended to become product key features able to provide supplementary benefits for the product developer and for the final user have been identified and some of them have been applied and analysed within the case study, for providing effectiveness and efficiency through the reconfigurable product development activities.

An innovative and flexible solution of a reconfigurable multi-station welding workbench, intended to solve the problem of layout optimisation, which usually most of the welding workshops have to deal with, has been designed and analysed within this work. Several benefits of that solution can be nominated: custom engineered to meet welding workshops facilities and application requirements; space optimization; low cost production and installation; modular design for ease of assembly, installation or rearranging; cleaner work environment.

Solid Edge parameterized modelling capabilities have enhanced the flexibility of the reconfigurable product model and have created premises for increasing the agility in technological changes implementation.

As another conclusion of the research approach, a few directions of further research development are identified for enhancing the Efficiency and Effectiveness of Product Development Process and the New Product Development Sustainability: integrating also an Economic Efficiency Analysis and a Life Cycle Impact Assessment approach as Concurrent Engineering activities will be considered.

\section{References}

1. A. Gunasekaran, Int. J. Prod. Res. 36, 5, 1223-1247 (1998)

2. B. Xing, G. Bright, N.S. Tlale, J. Potgieter, CAD/CAM, Robotics and Factories of the Future, 473 - 482 (2006)

3. A.S.Yigit, A.G. Ulsoy, A. Allahverdi, Journal of Intelligent Manufacturing 13, 309$316(2002)$

4. Y. Koren, M. Shpitalni, JMSY 29, 130-141 (2010)

5. V. Merticaru, M.I. Ripanu, M.A. Mihalache, M.M. Cucos, Applied Mechanics and Materials 809-810, 1492-1497 (2015)

6. V. Merticaru, G. Nagit, B. Pralea, R. Oana, AJME 12, $43-48$ (2014)

7. V. Merticaru, M.I. Ripanu, T.M. Runcanu, M.M. Nechita, S.C. Minciu, Applied Mechanics and Materials 657, 996-1000 (2014)

8. V. Merticaru, M.I. Ripanu, Applied Mechanics and Materials 371, 499-503 (2013)

9. E. Pelchat, A Brief Introduction to Structured Design (Coll. of Charleston, USA, 2004)

10. J. Gubau, Temes de Disseny 32, 57-71 (2016)

11. A.M. Del Corral, J. Fernández, M. González, X. Riudor, Temes de disseny 32, 81-97 (2016)

12. K. Scharwath, Yves Béhar's 7 Principles of Holistic Product Design (Triple Pundit, 2011)

13. A.C. Paraschiv, Bachelor Thesis (TUIASI, 2015)

14. SIEMENS, SolidEdge (2017) 